\title{
Treatment strategy for rod fractures following corrective fusion surgery in adult spinal deformity depends on symptoms and local alignment change
}

\author{
Yu Yamato, MD, PhD, ${ }^{1}$ Tomohiko Hasegawa, MD, PhD, ${ }^{1}$ Sho Kobayashi, MD, PhD, ${ }^{1}$ \\ Tatsuya Yasuda, MD, ${ }^{3}$ Daisuke Togawa, MD, PhD, ${ }^{1,2}$ Go Yoshida, MD, PhD, ${ }^{1}$ \\ Tomohiro Banno, MD, PhD, ${ }^{1}$ Shin Oe, MD, 1,2 Yuki Mihara, MD,1 and Yukihiro Matsuyama, MD, PhD1 \\ 1Department of Orthopaedic Surgery and ${ }^{2}$ Division of Geriatric Musculoskeletal Health, Hamamatsu University School of \\ Medicine; and ${ }^{3}$ Department of Orthopaedic Surgery, Hamamatsu Medical Center, Hamamatsu City, Shizuoka, Japan
}

OBJECTIVE Despite the significant incidence of rod fractures (RFs) following long-segment corrective fusion surgery, little is known about the optimal treatment strategy. The objectives of this study were to investigate the time course of clinical symptoms and treatments in patients with RFs following adult spinal deformity (ASD) surgery and to establish treatment recommendations.

METHODS This study was a retrospective case series of patients with RFs whose data were retrieved from a prospectively collected single-center database. The authors reviewed the cases of 304 patients (mean age 62.9 years) who underwent ASD surgery. Primary symptoms, time course of symptoms, and treatments were investigated by reviewing medical records. Standing whole-spine radiographs obtained before and after RF development and at last follow-up were evaluated. Osseous union was assessed using CT scans and intraoperative findings.

RESULTS There were 54 RFs in 53 patients (mean age 68.5 years [range 41-84 years]) occurring at a mean of 21 months (range 6-47 months) after surgery. In 1 patient RF occurred twice, with each case at a different time and level, and the symptoms and treatments for these 2 RFs were analyzed separately ( 1 case of revision surgery and 1 case of nonoperative treatment). The overall rate of RF observed on radiographs after a minimum follow-up of 1 year was $18.0 \%$ (54 of 300 cases). The clinical symptoms at the time of RF were pain in $77.8 \%$ (42 of 54 cases) and no onset of new symptoms in $20.5 \%$ (11 of 54 cases). The pain was temporary and had subsided in 19 of 42 cases (45\%) within 2 weeks. In 36 of the 54 cases $(66.7 \%$ ) (including the first RF in the patient with 2 RFs), patients underwent revision surgery at a mean of 116 days (range 5-888 days) after diagnosis. In 18 cases patients received only nonoperative treatment as of the last follow-up, including 17 cases in which the patients experienced no pain and no remarkable progression of deformity (mean 18.5 months after RF development).

CONCLUSIONS This analysis of 54 RFs in 53 patients following corrective fusion surgery for ASD demonstrates a relationship between symptoms and alignment change. Revision surgeries were performed in a total of 36 cases. Nonoperative care was offered in $18(33.3 \%)$ of 54 cases at the last follow-up, with no additional symptoms in 17 of the 18 cases. These data offer useful information regarding informed decision making for patients in whom an RF occurs after ASD surgery.

https://thejns.org/doi/abs/10.3171/2017.9.SPINE17525

KEYWORDS adult spinal deformity; complication; instrumentation failure; rod fracture; revision surgery; nonoperative care

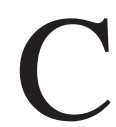
OMPARED with other chronic diseases, adult spinal deformity (ASD) has a particularly significant impact on quality of life. ${ }^{3,13}$ Accordingly, corrective fusion surgery for ASD has become more common, especially in elderly patients. Surgical procedures for ASD are generally challenging for spine surgeons due to the invasiveness of the surgery and the high rates of complications and reoperations..$^{4-6,10,14,15,21} \operatorname{Rod}$ fracture (RF) is a frequent implant-related complication following ASD surgery., $1,2,7,19$ RF may cause substantial pain and deterioration of spinal

ABBREVIATIONS 3-CO = 3-column osteotomy; $\mathrm{ASD}=$ adult spinal deformity; $\mathrm{C}$-CSVL = C-7 plum line and the center sacral vertical line; $\mathrm{PCO}=$ posterior column osteotomy; LL = lumbar lordosis; $\mathrm{PI}=$ pelvic incidence; PLF = posterolateral fusion; $\mathrm{PT}=$ pelvic tilt; $\mathrm{RF}=$ rod fracture; $\mathrm{SS}=\mathrm{sacral}$ slope; $\mathrm{SVA}=\mathrm{sagittal}$ vertical axis; $\mathrm{TK}=$ thoracic kyphosis.

SUBMITTED May 18, 2017. ACCEPTED September 26, 2017.

INCLUDE WHEN CITING Published online April 6, 2018; DOI: 10.3171/2017.9.SPINE17525. 
alignment, with a subsequent negative impact on clinical results and the mental health of patients. In one study, the incidence of implant-related complications was shown to be $31.7 \%$, and $52.6 \%$ of those patients required reoperation..$^{18}$ In the same study, rod breakage accounted for $47 \%$ of the implant-related complications. ${ }^{18}$ The incidence of RFs following ASD surgery has been reported by several authors: Smith et al. ${ }^{16}$ reported a $6.8 \%$ incidence of symptomatic rod breakage in patients who underwent ASD surgery. The same authors ${ }^{17}$ also reported development of an RF in $9.0 \%$ of an entire ASD patient population at a mean of 14.7 months and in $22.0 \%$ of patients who underwent pedicle subtraction osteotomy during a minimum 1-year follow-up. However, despite the high incidence of RFs, few reports have described the treatment methods and strategies for managing RFs. The development of RFs may cause symptoms such as low-back pain, buttock pain, and cracking sounds. Asymptomatic cases of RF can be found incidentally on routine radiography.,2,17 The time course of RF symptoms is heterogeneous. Pain diminishes immediately in some cases but persists in others. The objectives of this study were to investigate the time course of clinical symptoms and treatment progress in patients with RFs following ASD surgery and to establish treatment recommendations.

\section{Methods}

This study was approved by the institutional review board of the Hamamatsu University School of Medicine. Data were retrospectively collected from a single-center prospectively maintained database of ASD operations. ASD was defined as the presence of at least one of the following indicators: degenerative or idiopathic scoliosis, deformity with posttraumatic fractures or iatrogenic deformity with spinal curvature greater than $20^{\circ}$ in the coronal plane, sagittal vertical axis (SVA) greater than 50 $\mathrm{mm}$, pelvic tilt (PT) greater than $25^{\circ}$, and/or thoracic kyphosis (TK) greater than $60^{\circ}$. Included in this study were consecutive ASD patients aged 18 years or older, treated between May 2010 and December 2015, in whom corrective fusion surgery involved a minimum of 3 levels of fusion. An additional inclusion criterion was the need for whole-spine radiographs obtained at a minimum of 1 year after primary surgery. Patients who underwent surgeries for cervical deformity or traumatic fracture in the acute phase were excluded. RFs were defined as breakage of the rod identified using radiographs regardless of the presence or absence of symptoms, which included fractures incidentally detected on routine examination of radiographic images. The primary symptoms and the time course of symptoms and treatment were carefully investigated by reviewing patient medical records. Our fundamental treatment approach to RFs was dependent on the patients' request. We presented 2 options-revision surgery and nonoperative care-to the patients with RFs. We also informed the patients that evidence of clinical results was not available. We did not urge patients to undergo revision surgery. The progressive loss of correction and the development of symptoms were considered in relation to the initial symptoms.

\section{Symptoms of RF Development}

Patients with an RF were classified into 4 groups depending on their primary symptoms: asymptomatic, temporary pain (pain diminished within 2 weeks of diagnosis), continuous pain (pain continued for more than 2 weeks), and neural symptoms. The temporary pain group included those who heard a cracking sound in their back.

\section{Image Findings}

We obtained posteroanterior and lateral whole-spine radiographs with patients in the standing position before and after RF development and at the last follow-up. The parameters measured on the standing whole-spine radiographs were as follows: the distance between the C-7 plum line and the center sacral vertical line (C7-CSVL) in the frontal views and the SVA, lumbar lordosis (LL), TK, PT, sacral slope (SS), pelvic incidence (PI), PI-LL, and local angle (angle between the upper endplate of the proximal vertebra and the lower endplate of the distal vertebra around the RF site) on the lateral views. Osseous healing of the anterior spinal column was assessed using 3D CT scans after RF development. A total of 4 sites-upper and lower connection sites between the endplate or surface of the vertebral osteotomy and grafted bone on both coronal and sagittal images-were used to evaluate osseous healing at the 3-column osteotomy (3-CO) and interbody fusion levels. Osseous union was defined as arthrodesis in all 4 sites.

\section{Intraoperative Findings}

Osseous healing was also evaluated during revision surgery. The mobility of the fused segment while applying direct passive stress to the screw was evaluated with the rod removed. We defined osseous nonunion as obvious mobility at the RF site.

\section{Statistical Analysis}

Descriptive statistics, including means and standard deviations, were calculated for demographic data. Statistical analysis was performed using 1-way ANOVA, followed by Tukey's post hoc test and paired t-tests. A p $<0.05$ was considered significant. Predictor variables for the presence of continuous pain were analyzed using single and multiple regression analysis. All statistical computations were performed using IBM SPSS software (version 21.0; IBM Corp.).

\section{Results}

\section{Demographics of All Patients With RFs}

Data obtained in 304 consecutively treated ASD patients (mean age 62.9 years) were reviewed (Table 1). Whole-spine radiographs obtained at a minimum of 1 year after surgery were available for 300 patients (followup rate $98.7 \%$ ). There were $54 \mathrm{RFs}$ in 53 patients (43 females, 10 males; mean age 68.5 years [range $41-84$ years]) occurring at a mean of 21 months (range 6-47 months) after surgery. RF occurred twice at different times and levels in a 74-year-old female patient. Because the main purpose of this paper was the analysis of time course of clinical symptoms and treatment, the symptoms and treat- 
TABLE 1. Demographic data

\begin{tabular}{|c|c|c|c|c|c|}
\hline \multirow[b]{2}{*}{ Demographic \& RF Characteristics } & \multicolumn{5}{|c|}{ Symptoms } \\
\hline & Asymptomatic & Temporary Pain & Continuous Pain & Neural & Total $(\%)$ \\
\hline No. of cases & 11 & 19 & 23 & 1 & 54 \\
\hline Mean age (yrs) & 70.5 & 66.6 & 69.4 & 63.0 & \\
\hline F/M ratio & $7: 4$ & $17: 2$ & $18: 5$ & $1: 0$ & \\
\hline Mean duration btwn op \& RF (mos) & 23.5 & 23.5 & 17.4 & 28.0 & \\
\hline Mean no. of fusion levels & 9.1 & 9.3 & 10.3 & 10.0 & \\
\hline \multicolumn{6}{|l|}{ RF site } \\
\hline 3-CO & 2 & 7 & 7 & 1 & $17(31.5)$ \\
\hline PCO & 3 & 8 & 9 & 0 & $20(37.0)$ \\
\hline PLF & 3 & 2 & 6 & 0 & $11(20.4)$ \\
\hline LLIF & 0 & 0 & 1 & 0 & $1(1.9)$ \\
\hline Sacroiliac joint & 3 & 2 & 0 & 0 & $5(9.3)$ \\
\hline \multicolumn{6}{|l|}{ RF levels (except 3-CO) } \\
\hline $\mathrm{T} 12-\mathrm{L} 1$ & 0 & 1 & 0 & 0 & 1 \\
\hline L1-2 & 0 & 0 & 1 & 0 & 1 \\
\hline L3-4 & 0 & 1 & 3 & 0 & 4 \\
\hline L4-5 & 1 & 3 & 5 & 0 & 9 \\
\hline L5-S1 & 5 & 5 & 7 & 0 & 17 \\
\hline Sacrum/ilium & 3 & 2 & 0 & 0 & 5 \\
\hline \multicolumn{6}{|l|}{ Rod composition/diameter (mm) } \\
\hline Titanium alloy/6.0 & 4 & 3 & 3 & 0 & $10(18.5)$ \\
\hline Titanium alloy/6.35 & 2 & 5 & 8 & 0 & $15(27.8)$ \\
\hline Cobalt chromium/6.0 & 0 & 3 & 1 & 1 & $5(9.3)$ \\
\hline Commercially pure titanium/6.0 & 4 & 5 & 5 & 0 & $14(25.9)$ \\
\hline Commercially pure titanium/6.35 & 1 & 3 & 6 & 0 & $10(18.5)$ \\
\hline \multicolumn{6}{|l|}{ Side of fractured rod } \\
\hline Unilateral & 5 & 9 & 3 & 0 & $17(31.5)$ \\
\hline Unilateral $\rightarrow$ bilateral & 3 & 6 & 11 & 1 & $21(38.9)$ \\
\hline Bilateral & 3 & 4 & 9 & 0 & $16(29.6)$ \\
\hline
\end{tabular}

$\mathrm{LLIF}=$ lateral lumbar interbody fusion; unilateral $\rightarrow$ bilateral = fracture was initially unilateral but developed into a bilateral fracture.

ments for these $2 \mathrm{RF}$ cases were analyzed separately. The incidence rate of RFs was $18.0 \%$ (54 of 300 cases). The mean follow-up period after ASD surgery in patients who sustained an RF was 42.4 months (range 16-62 months).

The upper instrumented vertebra in the 53 patients with RFs was T- 3 in 1 patient, T- 4 in 7 patients, T- 5 in 2 patients, T-6 in 1 patient, T-7 in 2 patients, T-8 in 6 patients, T-9 in 7 patients, and T-10 in 27 patients. The lower instrumented vertebra was the ilium in all patients. RF occurred at the site of the 3-CO in 17 cases, posterior column osteotomy (PCO) in 20 cases, posterolateral fusion (PLF) in 11 cases, lateral lumbar interbody fusion in 1 case, and sacroiliac joint in 5 cases. RF levels (except in cases of 3-CO) were T12-L1 in 1 case, L1-2 in 1 case, L3-4 in 4 cases, L4-5 in 9 cases, L5-S1 in 17 cases, and sacroiliac joint in 5 cases. Rod composition and diameter were as follows: titanium alloy $(6.0 \mathrm{~mm}, 10$ cases; $6.35 \mathrm{~mm}, 15$ cases), cobalt chromium $(6.0 \mathrm{~mm}, 5$ cases$)$, and commercially pure titanium (6.0 mm, 14 cases; $6.35 \mathrm{~mm}, 10$ cases).

RFs initially occurred in a unilateral rod in 38 cases and bilateral rods in 16 cases. In 21 of the 38 initial uni- lateral RF cases, RFs developed in the bilateral rods during follow-up. The number of rods in the primary ASD surgery was 2 in 46 cases, 3 in 7 cases, and 4 in 1 case. All RFs occurred at sites with 2 rods.

\section{Symptoms of RF}

The clinical symptoms at the time of RF were additional back, low-back, or buttock pain in 42 cases (77.8\%) (Table 1). In 14 cases, patients heard cracking sounds in their backs. The pain diminished within 2 weeks of RF in 19 of $42(45 \%)$ cases. Lower-extremity pain developed in 1 case. In the remaining 11 cases there was no onset of new symptoms, and RFs were incidentally diagnosed on radiographs at a follow-up visit. Patients with RFs were classified as being asymptomatic in 11 cases (including the second RF in the patient with 2 RF cases), having temporary pain in 19 cases, having continuous pain in 23 cases (including the first RF in the patient with $2 \mathrm{RF}$ cases), and having neural symptoms in 1 case. There were no significant differences in age, sex, and interval from ASD 
TABLE 2. Treatment progress in cases initially treated with nonoperative care

\begin{tabular}{|c|c|c|c|c|c|c|}
\hline \multirow[b]{2}{*}{ Initial Plan } & \multirow[b]{2}{*}{ Treatment at Final FU } & \multicolumn{4}{|c|}{ Symptoms } & \multirow[b]{2}{*}{ Total } \\
\hline & & Asymptomatic & Temporary Pain & Continuous Pain & Neural & \\
\hline Nonoperative care & & 10 & 10 & 7 & 0 & 27 \\
\hline & Continuation of nonop & 10 & 7 & 1 & & 18 \\
\hline & Conversion to revision op & 0 & 3 & 6 & & 9 \\
\hline Revision op & & 1 & 9 & 16 & 1 & 27 \\
\hline
\end{tabular}

$\mathrm{FU}=$ follow-up

Data are presented as number of cases.
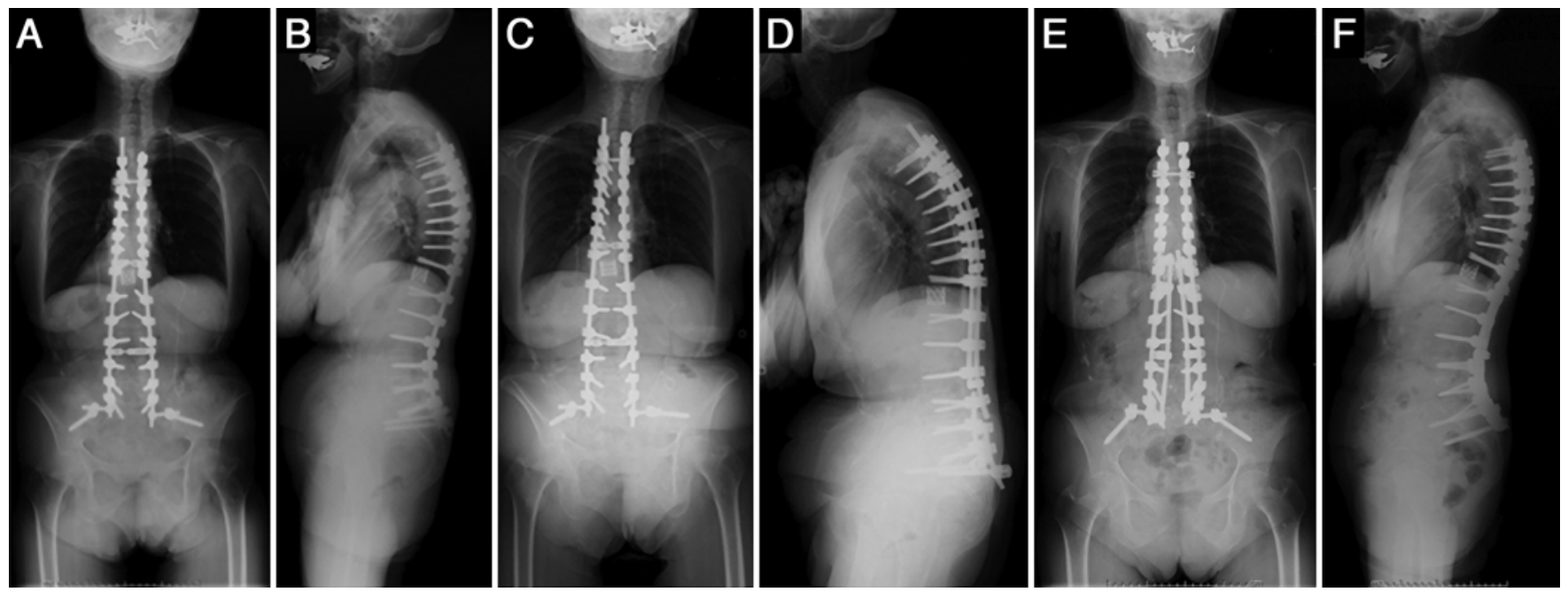

FIG. 1. Case example of RF with continuous pain. The patient is a 71-year-old woman with kyphosis that developed following a T-12 vertebral fracture. A and B: Posterior corrective T5-ilium fusion with vertebral column resection was performed. $\mathbf{C}$ and $\mathbf{D}$ : RF at $L 4-5$ with continuous back pain developed at 35 months after primary surgery. $E$ and $F$ : Revision surgery was performed to replace the rod with 2 additional rods, and interbody fusion was performed at L4-5 and L5-S1.

surgery to RF among patients who were asymptomatic, had temporary pain, or had continuous pain. The characteristics of the RF site, RF level, and RF side were not statistically analyzed because of the small number of cases.

\section{Treatment Progress of RF}

In 27 cases, the patients initially received nonoperative care in the form of a thoracolumbar orthosis worn for 3 months (Table 2). As of the last follow-up, treatment was converted to revision surgery in 9 of these 27 cases. In 36 cases patients underwent revision surgery at a mean of 116 days after diagnosis (range 5-888 days). In 10 of the 11 asymptomatic cases (including the second RF in the patient with $2 \mathrm{RFs}$ ), patients chose nonoperative care after RF development, and in no case was this converted to revision surgery at the final follow-up. In the 19 cases with temporary pain after RF, nonoperative care was chosen by 10 patients and revision surgery by 9 patients. Of the 10 conservatively managed cases in the patients with temporary pain, 3 were ultimately converted to revision surgery because increased pain and discomfort developed. In the cases of continuous pain, 7 patients initially requested nonoperative care, and in 6 of the 7 cases treatment was converted to revision surgery. In the remaining case the patient with continuous pain chose not to undergo revision surgery.

In all cases of revision surgery, patients underwent replacement of the bilateral rods, either the entire rod or a portion of the rod with the use of connectors, accompanied by satellite rods at all lumbar levels., ${ }^{912}$ Interbody fusions were part of the revision surgery in 12 cases, including transforaminal lumbar interbody fusion at the PCO and PLF sites. The compression force was adapted and PLF was performed at 3-CO sites with RFs. Symptoms improved in all patients after revision surgery (Fig. 1).

Nonoperative care was provided in 18 cases at the last follow-up. During the follow-up period (mean 18.5 months [range 7-33 months] after RF), patients were asymptomatic in 17 of the 18 cases (Fig. 2).

Patients with RFs were classified into 2 groups according to the duration between their initial operation and when RF developed, either within or after 24 months, including the first case within 24 months and the second case after 24 months in the patient with 2RFs. We compared the incidences of revision surgery, symptoms, and alignment change between the 2 groups. The rate of revision surgery at the final follow-up was $76.3 \%$ (29 of 38 cases) in the group in which fractures occurred within the 

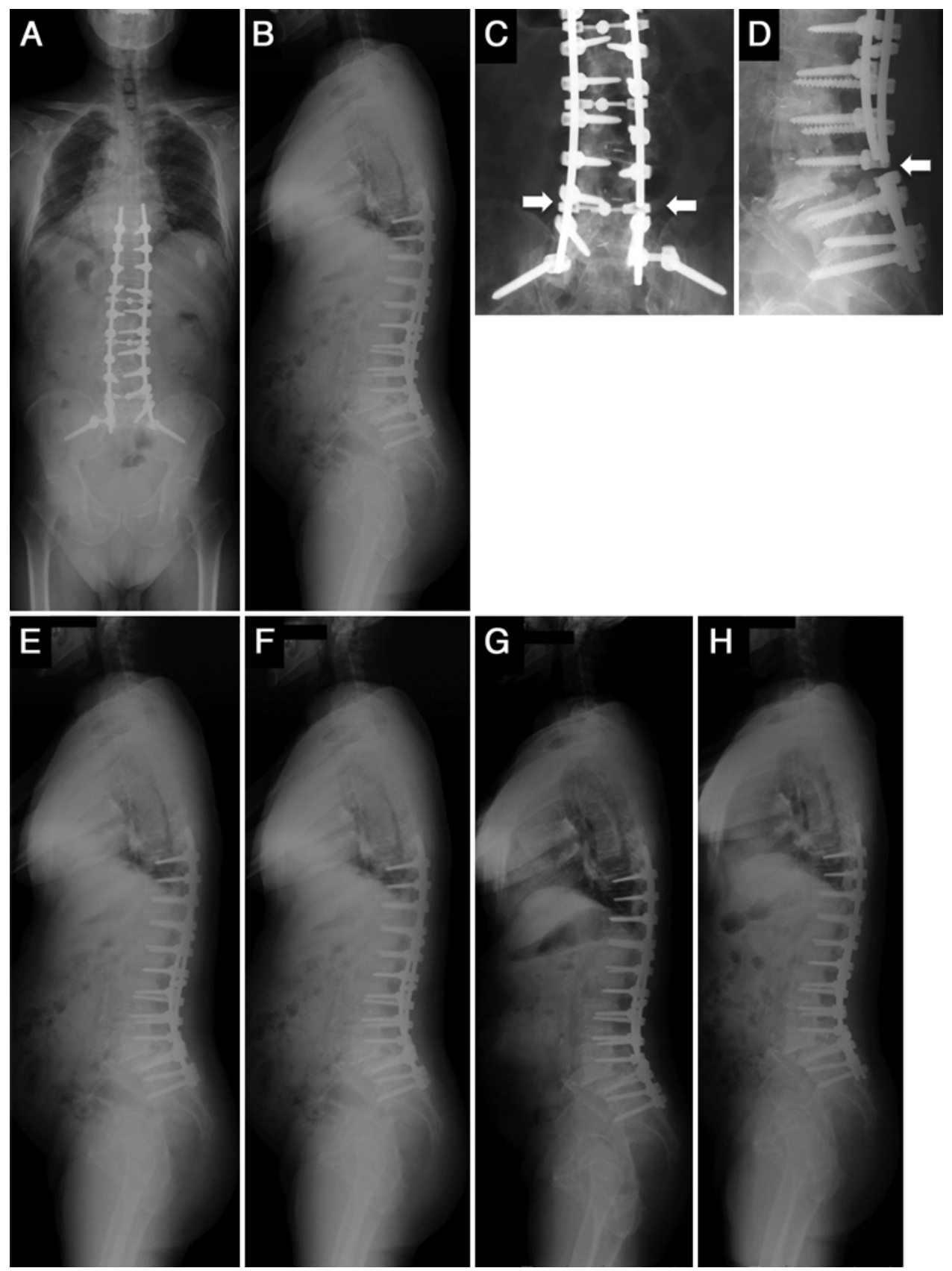

FIG. 2. Case example of asymptomatic RF. The patient is an 80 -year-old man with kyphoscoliosis. A and B: Posterior corrective fusion with multiple PCOs and interbody fusion was performed as the primary operation. C and D: Twenty-four months later, an RF was incidentally found on radiographs. There was no alignment change and no symptoms after the RF. E-H: Radiographs obtained before $R F(E)$, at diagnosis of $R F(F), 1$ year after $R F(G)$, and 3 years after $R F(H)$.

first 24 months and $43.8 \%$ (7 of 16 cases) in the group in which fractures occurred after 24 months $(\mathrm{p}<0.05)$. A significant difference was observed only in the incidence of revision surgery between the 2 groups.

\section{Radiographic Parameters}

Whole-spine standing radiographs obtained before and after RFs were examined in 46 cases (10 with no symptoms [including the second case in the patient with $2 \mathrm{RFs}$ ],
18 with temporary pain, and 18 with continuous pain [including the first case in the patient with 2 RFs]) (Table 3 ). In the remaining 8 cases, 7 patients could not endure radiographic imaging in the standing position due to their pain and 1 asymptomatic patient had radiographs taken only in the supine position. No radiographic parameters noted prior to the RFs were significantly different among the 3 groups. A significant difference was observed in the PT (asymptomatic vs continuous pain groups) and local 
TABLE 3. Spinopelvic parameters

\begin{tabular}{|c|c|c|c|c|c|}
\hline Parameter & Asymptomatic & Temporary Pain & Continuous Pain & $p$ Value & Post Hoc Test \\
\hline No. of cases & 10 & 18 & 18 & & \\
\hline \multicolumn{6}{|c|}{ C7-CSVL (mm) } \\
\hline Pre-RF & $25.3 \pm 37.4$ & $19.3 \pm 14.0$ & $20.2 \pm 12.2$ & 0.748 & \\
\hline Post-RF & $27.3 \pm 28.3$ & $24.3 \pm 20.6$ & $24.1 \pm 16.3$ & 0.918 & \\
\hline Variation & $2.0 \pm 8.4$ & $5.0 \pm 6.2$ & $3.9 \pm 5.8$ & 0.665 & \\
\hline \multicolumn{6}{|l|}{ SVA (mm) } \\
\hline Pre-RF & $54.0 \pm 67.9$ & $51.6 \pm 61.8$ & $57.6 \pm 51.3$ & 0.954 & \\
\hline Post-RF & $75.0 \pm 62.3$ & $71.2 \pm 68.3$ & $105.4 \pm 75.2$ & 0.306 & \\
\hline Variation & $21.0 \pm 21.2$ & $19.7 \pm 31.8$ & $47.8 \pm 42.7$ & 0.041 & NS \\
\hline \multicolumn{6}{|l|}{$\operatorname{LL}\left({ }^{\circ}\right)$} \\
\hline Pre-RF & $45.7 \pm 13.8$ & $41.9 \pm 9.9$ & $44.4 \pm 10.3$ & 0.65 & \\
\hline Post-RF & $41.9 \pm 11.8$ & $38.8 \pm 10.2$ & $31.9 \pm 15.4$ & 0.11 & \\
\hline Variation & $-3.8 \pm 6.4$ & $-3.2 \pm 6.0$ & $-12.6 \pm 9.3$ & 0.001 & $A S$ vs $C P, T P$ vs $C P$ \\
\hline \multicolumn{6}{|l|}{$\operatorname{TK}\left({ }^{\circ}\right)$} \\
\hline Pre-RF & $42.6 \pm 14.5$ & $42.5 \pm 14.2$ & $47.3 \pm 14.5$ & 0.556 & \\
\hline Post-RF & $44.7 \pm 15.2$ & $42.4 \pm 14.9$ & $47.6 \pm 15.2$ & 0.597 & \\
\hline Variation & $2.1 \pm 7.3$ & $-0.1 \pm 6.0$ & $-2.3 \pm 16.9$ & 0.633 & \\
\hline \multicolumn{6}{|l|}{$\mathrm{PT}\left({ }^{\circ}\right)$} \\
\hline Pre-RF & $20.4 \pm 8.1$ & $25.0 \pm 7.4$ & $24.7 \pm 7.9$ & 0.288 & \\
\hline Post-RF & $20.8 \pm 7.9$ & $26.9 \pm 7.8$ & $30.0 \pm 10.4$ & 0.042 & AS vs CP \\
\hline Variation & $0.4 \pm 3.2$ & $1.9 \pm 3.5$ & $5.3 \pm 5.3$ & 0.011 & AS vs $C P$ \\
\hline \multicolumn{6}{|l|}{$\operatorname{SS}\left({ }^{\circ}\right)$} \\
\hline Pre-RF & $30.1 \pm 12.7$ & $28.1 \pm 8.7$ & $28.1 \pm 9.0$ & 0.846 & \\
\hline Post-RF & $29.4 \pm 11.1$ & $26.5 \pm 9.0$ & $22.8 \pm 9.7$ & 0.218 & \\
\hline Variation & $-0.7 \pm 4.5$ & $-1.6 \pm 3.9$ & $-5.3 \pm 6.4$ & 0.037 & NS \\
\hline \multicolumn{6}{|l|}{$\mathrm{PI}\left({ }^{\circ}\right)$} \\
\hline Pre-RF & $50.5 \pm 10.4$ & $53.1 \pm 10.2$ & $52.8 \pm 11.4$ & 0.819 & \\
\hline Post-RF & $50.2 \pm 11.2$ & $53.4 \pm 10.9$ & $52.8 \pm 10.8$ & 0.746 & \\
\hline Variation & $-0.3 \pm 4.8$ & $0.4 \pm 2.7$ & $0.0 \pm 2.7$ & 0.859 & \\
\hline \multicolumn{6}{|l|}{$\mathrm{PI}-\mathrm{LL}\left({ }^{\circ}\right)$} \\
\hline Pre-RF & $4.8 \pm 10.3$ & $11.1 \pm 11.6$ & $8.3 \pm 10.7$ & 0.11 & \\
\hline Post-RF & $8.3 \pm 11.9$ & $14.7 \pm 13.9$ & $20.9 \pm 16.4$ & 0.097 & \\
\hline Variation & $3.5 \pm 5.2$ & $3.6 \pm 6.5$ & $12.6 \pm 8.3$ & 0.001 & AS vs $C P, T P$ vs $C P$ \\
\hline \multicolumn{6}{|l|}{ Local angle $\left(^{\circ}\right)$} \\
\hline Pre-RF & $25.6 \pm 13.1$ & $23.4 \pm 14.5$ & $18.7 \pm 8.4$ & 0.171 & \\
\hline Post-RF & $22.7 \pm 12.5$ & $21.2 \pm 14.4$ & $8.9 \pm 16.4$ & 0.012 & AS vs $C P, T P$ vs $C P$ \\
\hline Variation & $-2.9 \pm 3.7$ & $-2.2 \pm 3.6$ & $-9.7 \pm 8.3$ & 0.001 & AS vs $C P, T P$ vs $C P$ \\
\hline
\end{tabular}

$\mathrm{AS}=$ asymptomatic; $\mathrm{CP}=$ continuous pain; $\mathrm{NS}=$ not significant; $\mathrm{TP}=$ temporary pain.

Mean values are presented \pm SD. Boldface type indicates statistical significance.

angle (asymptomatic vs continuous pain, temporary pain vs continuous pain) on post-RF radiographs. The amount of change after RF was significantly different in the LL (asymptomatic and temporary pain vs continuous pain), PT (asymptomatic vs continuous pain), PI-LL (asymptomatic and temporary pain vs continuous pain), and local angle (asymptomatic and temporary pain vs continuous pain).

Nonoperative care was initially provided in 27 cases. In 9 of these cases the patients ultimately elected to undergo revision surgery during the follow-up period. Table
4 shows the radiographic parameters of the cases in which patients continued with nonoperative care or converted to revision surgery. There was no difference between the 2 groups before RF development. The number of changes after RF in patients who converted to revision surgery was significantly greater than the number in patients continuing with nonoperative care in terms of LL $(p=0.006)$, PT $(\mathrm{p}=0.035)$, PI-LL $(\mathrm{p}=0.001)$, and local angle $(\mathrm{p}=0.014)$. In 1 case, continuous pain was present, and in the other 17 cases (31.5\%) no additional symptoms of RF developed. 
TABLE 4. Alignment comparison between continuation of conservative therapy and conversion to revision surgery

\begin{tabular}{|c|c|c|c|}
\hline Parameter & $\begin{array}{l}\text { Continuation of } \\
\text { Nonop Care }\end{array}$ & $\begin{array}{l}\text { Conversion to } \\
\text { Revision Op }\end{array}$ & $p$ Value \\
\hline No. of cases & 18 & 9 & \\
\hline \multicolumn{4}{|c|}{ C7-CSVL (mm) } \\
\hline Pre-RF & $24.2 \pm 29.5$ & $17.3 \pm 8.3$ & 0.505 \\
\hline Post-RF & $27.5 \pm 24.9$ & $22.9 \pm 18.2$ & 0.631 \\
\hline Variation & $3.2 \pm 15.7$ & $5.6 \pm 18.1$ & 0.743 \\
\hline \multicolumn{4}{|l|}{ SVA (mm) } \\
\hline Pre-RF & $55.1 \pm 67.7$ & $46.4 \pm 32.5$ & 0.722 \\
\hline Post-RF & $74.3 \pm 68.1$ & $89.7 \pm 49.4$ & 0.556 \\
\hline Variation & $19.2 \pm 30.2$ & $43.2 \pm 36.0$ & 0.083 \\
\hline \multicolumn{4}{|l|}{$\operatorname{LL}\left({ }^{\circ}\right)$} \\
\hline Pre-RF & $46.5 \pm 12.3$ & $43.6 \pm 8.8$ & 0.537 \\
\hline Post-RF & $43.6 \pm 10.5$ & $32.0 \pm 8.7$ & 0.009 \\
\hline Variation & $-2.9 \pm 5.7$ & $-11.6 \pm 8.8$ & 0.006 \\
\hline \multicolumn{4}{|l|}{$\operatorname{TK}\left({ }^{\circ}\right)$} \\
\hline Pre-RF & $47.6 \pm 13.6$ & $46.8 \pm 11.0$ & 0.881 \\
\hline Post-RF & $48.6 \pm 12.6$ & $48.3 \pm 15.0$ & 0.949 \\
\hline Variation & $1.1 \pm 8.9$ & $-3.9 \pm 22.8$ & 0.433 \\
\hline \multicolumn{4}{|l|}{ PT $\left(^{\circ}\right)$} \\
\hline Pre-RF & $21.2 \pm 8.4$ & $25.7 \pm 6.7$ & 0.177 \\
\hline Post-RF & $22.9 \pm 8.9$ & $30.9 \pm 8.8$ & 0.039 \\
\hline Variation & $1.7 \pm 3.5$ & $5.2 \pm 4.4$ & 0.035 \\
\hline \multicolumn{4}{|l|}{$\operatorname{SS}\left({ }^{\circ}\right)$} \\
\hline Pre-RF & $30.8 \pm 9.0$ & $24.1 \pm 9.3$ & 0.085 \\
\hline Post-RF & $29.5 \pm 8.5$ & $19.7 \pm 10.2$ & 0.015 \\
\hline Variation & $-1.3 \pm 4.5$ & $-4.4 \pm 6.6$ & 0.164 \\
\hline \multicolumn{4}{|l|}{$\mathrm{PI}\left({ }^{\circ}\right)$} \\
\hline Pre-RF & $52.0 \pm 8.9$ & $49.8 \pm 11.0$ & 0.582 \\
\hline Post-RF & $52.4 \pm 9.6$ & $50.6 \pm 11.3$ & 0.662 \\
\hline Variation & $0.4 \pm 4.1$ & $0.8 \pm 3.4$ & 0.822 \\
\hline \multicolumn{4}{|l|}{ PI-LL $\left(^{\circ}\right)$} \\
\hline Pre-RF & $5.5 \pm 12.4$ & $6.2 \pm 10.9$ & 0.889 \\
\hline Post-RF & $8.8 \pm 13.4$ & $18.6 \pm 13.6$ & 0.093 \\
\hline Variation & $3.3 \pm 4.9$ & $12.3 \pm 7.3$ & 0.001 \\
\hline \multicolumn{4}{|c|}{ Local angle $\left(^{\circ}\right)$} \\
\hline Pre-RF & $25.8 \pm 14.6$ & $18.2 \pm 7.6$ & 0.163 \\
\hline Post-RF & $23.2 \pm 14.9$ & $11.0 \pm 12.3$ & 0.048 \\
\hline Variation & $-1.1 \pm 3.6$ & $-7.2 \pm 8.3$ & 0.014 \\
\hline
\end{tabular}

Mean values are presented $\pm S D$. Boldface type indicates statistical significance.

Table 5 shows radiographic parameters of the 17 cases with no additional symptoms who underwent nonoperative care at the final follow-up (mean 18.5 months after RF development). Only the parameter C7-CSVL was significantly improved at the final follow-up compared with RF development $(\mathrm{p}=0.032)$.

CT scanning was performed following RF in 35 cases. Osseous healing was assessed in 15 of 35 cases in which
TABLE 5. Alignment after RF and at final follow-up in 17 cases with nonoperative treatment and with no additional symptoms

\begin{tabular}{lccc}
\hline \multicolumn{1}{c}{ Parameter } & Post-RF & Final FU & p Value \\
\hline C7-CSVL $(\mathrm{mm})$ & $27.5 \pm 25.5$ & $21.8 \pm 25.3$ & 0.032 \\
\hline SVA $(\mathrm{mm})$ & $77.3 \pm 64.7$ & $84.0 \pm 68.5$ & 0.486 \\
\hline $\mathrm{LL}\left({ }^{\circ}\right)$ & $37.8 \pm 21.5$ & $42.3 \pm 11.1$ & 0.806 \\
\hline $\mathrm{TK}\left({ }^{\circ}\right)$ & $49.0 \pm 13.0$ & $47.4 \pm 13.1$ & 0.323 \\
\hline $\mathrm{PT}\left({ }^{\circ}\right)$ & $23.7 \pm 10.4$ & $22.9 \pm 9.0$ & 0.505 \\
\hline $\mathrm{SS}\left({ }^{\circ}\right)$ & $29.1 \pm 8.5$ & $29.9 \pm 7.8$ & 0.695 \\
\hline $\mathrm{PI}\left({ }^{\circ}\right)$ & $50.1 \pm 11.6$ & $52.7 \pm 9.2$ & 0.408 \\
\hline $\mathrm{PI}-\mathrm{LL}\left({ }^{\circ}\right)$ & $11.6 \pm 16.1$ & $10.5 \pm 13.2$ & 0.135 \\
\hline Local angle $\left(^{\circ}\right)$ & $22.4 \pm 16.8$ & $25.0 \pm 17.1$ & 0.624 \\
\hline
\end{tabular}

Mean values are presented \pm SD. Boldface type indicates statistical significance.

either 3-CO or PCO with interbody fusion was performed. Intraoperative findings indicated physical motion at the RF site in 22 of the 36 patients; no sign of motion at the RF site during revision surgery was observed in the remaining 14 patients. Comparing mobility at revision surgery to osseous healing on CT scans in 29 cases, osseous healing with no mobility at the revision surgery was seen in 5 cases and the absence of osseous healing with the presence of mobility was seen in 15 . In the remaining 9 cases, a discrepancy between image findings and surgical findings was found (Table 6).

\section{Predictor Variables of Continuous Pain}

Variables predictive of the presence of continuous pain were analyzed using single and multiple regression analyses. In single regression analysis, the predictor variables were the extents of change in the LL, PT, SS, PI-LL, local angle, and RF periods $(\mathrm{p}<0.05)$. Incomplete union shown on CT scans was not a predictor. The multiple regression analysis revealed that the only predictor for continuous pain was the extent of change in PI-LL (OR 1.198, 95\% CI $1.056-1.359)(\mathrm{p}=0.005)$.

\section{Discussion}

Previous studies have shown a high incidence (range $6.8 \%-22 \%$ ) of RFs following corrective fusion surgery in patients treated for ASD. ${ }^{1,2,16,17,19}$ In the present study, the overall radiographic rate of RFs in patients with a minimum of 1 year of follow-up was $18.0 \%$. RFs developed at a mean of 21 months (range 6-47 months) after primary surgery, and a higher incidence of RFs would be expected with time. However, despite their significantly high incidence, the symptoms, time course, and outcomes of RF are not well documented. The present study provides a detailed investigation of symptom and treatment progress after RFs in 54 cases of RF in 53 patients.

In some previously reported studies, the authors investigated the symptoms and progression of RFs. Smith et al. ${ }^{16}$ reported that pain was the primary symptom in $97 \%$ (29 of 30) of patients, with symptomatic RF and postoperative loss of deformity correction noted in 17\%. In a different re- 
TABLE 6. Comparison between CT findings and intraoperative findings in 29 cases

\begin{tabular}{lcccc}
\hline \multirow{2}{*}{ Finding } & & \multicolumn{3}{c}{ Mobility in Revision Op } \\
\cline { 3 - 4 } & Present & Yes & No & Total \\
\hline \multirow{2}{*}{ Osseous contact on CT } & Yes & 5 & 6 & 11 \\
\cline { 2 - 5 } & No & 3 & 15 & 18 \\
\hline \multirow{2}{*}{ Total } & & 8 & 21 & 29 \\
\hline
\end{tabular}

Data are presented as number of cases.

port from Smith et al., ${ }^{17}$ incidental fractures were found on routine imaging in 6 of $18 \mathrm{RF}$ patients, and there were no apparent clinical symptoms. Akazawa et al. ${ }^{1}$ reported the following clinical symptoms at the time of fracture: pain in 2 patients and cracking sounds in 2 patients; no symptoms were noted in 4 patients. However, the time courses of symptoms were not addressed in these reports. Our study revealed that the clinical symptoms at the time of RFs were pain in $42(77.8 \%)$ of 54 cases, neural symptoms in 1 case $(1.9 \%)$, and no onset of new symptoms in 11 cases $(20.4 \%)$. In cases with pain, however, the pain had resolved in $19(45 \%)$ of 42 cases within 2 weeks. Our findings of either no pain or resolution of pain in $56 \%(30 / 54)$ of RF cases within 2 weeks of RF indicate that the development of an RF may not have a significant impact on the patient during clinical evaluation.

The other main problem caused by RFs is loss of correction. Few reports have mentioned that loss of correction and changes in alignment occur following RF. Alignment change before and after RF were analyzed in the present study (Table 3), and we observed a relationship between symptoms and changes in alignment. Pain continued in the case of severe correction loss at the RF site. Conversely, only slight correction loss was observed in asymptomatic patients. Of the patients in this study who initially elected nonoperative care, conversion to revision surgery was ultimately seen in 33.3\% (9/27) because pain and discomfort progressed. Significant deterioration in sagittal alignment correction was observed in patients in whom conversion to surgery was performed. Loss of correction and alignment deterioration were an indicator that revision surgery was necessary.

The treatment approach for RFs varies by surgeons and reports. Smith et al. ${ }^{16}$ reported that $87 \%$ of symptomatic RF patients had undergone revision surgery at the last follow-up and the remaining patients had experienced an improvement in symptoms with nonoperative treatments and thus declined revision surgery. Smith et al. ${ }^{17}$ also reported that 12 of 18 patients with RFs had undergone revision surgery, primarily consisting of rod replacement and repeat arthrodesis. In contrast, some authors have performed revision surgery in all RF patients regardless of the presence of symptoms. ${ }^{1,11}$ In the current study, nonoperative care was provided in $33.3 \%$ (18/54) of cases at the last follow-up, with no additional symptoms and little change in alignment in 17 of the 18 cases. In such cases, spinal alignment in the standing position was maintained after RF. The results of this study suggest some guidance toward an informed choice. In the asymptomatic group, none of the patients required revision surgery. Nonoperative care is reliably indicated for asymptomatic patients with an RF. In our continuous pain group, revision surgery was performed in 22 of 23 cases at the final follow-up. Patients with continuous pain were advised to undergo revision surgery. The optimum therapy for patients with diminished pain is a matter of debate; in 3 of 10 cases in the present series, treatment was converted to revision surgery. When patients choose nonoperative care, close follow-up should be provided. Progressive loss of correction may make revision surgery difficult and complicated; therefore, it is important to ascertain whether correction loss has occurred at the RF site. This study revealed no deterioration of local alignment in patients without additional symptoms (Table 5). This data point may be useful for determining which fractures must be repaired surgically.

In the study reported by Smith et al., ${ }^{17}$ pseudoarthrodesis was confirmed intraoperatively in all patients who underwent revision surgery. Luca et al. ${ }^{12}$ described posteroanterior combination surgery for achieving spinal fusion of the anterior column in revision surgery for pedicle subtraction osteotomy. In the present study no signs of mobility at the RF site were observed in 8 of 29 patients undergoing revision surgery. In cases with no sign of mobility at the RF site, it may be possible to achieve bone union using only posterior revision. It is necessary to assess bone union of the anterior column when the revision surgery method is determined. This study revealed the difficulty of making a precise assessment of bone union using image examinations. Fracture mechanisms of rods were analyzed using an emission scanning electron microscope, showing that the fatigue cracking occurred during rod use, whereas external stress eventually caused the rods to fracture. ${ }^{20}$ The construction of rods to disperse mechanical stress using accessory rods should be considered in revision surgery. ${ }^{9}$

This study has some limitations, including its retrospective design, single-center setting, short periods of follow-up, no clinical evaluation, and lack of a precise assessment of fusion status in all patients. Longer follow-up is certainly important for the investigation of nonoperative care after RF. Clinical evaluation after treatment of RF was not performed in all patients. It is important to clinically assess the outcomes of nonoperative or surgical RF treatment. Optimal assessment of spinal fusion is essential to prevent the recurrence of RF and determine the revision procedure. The present study revealed the discrepancies between CT and intraoperative findings in terms of fusion status and underlined the difficulty in assessing bone fusion. A method for the precise assessment of bone union is required to effectively treat $\mathrm{RF}$ in patients with long spinal fusions.

\section{Conclusions}

Our analysis of 54 RFs in 53 patients following corrective fusion surgery for ASD showed the relationship between symptom and alignment change. Revision surgeries were performed in a total of 36 cases. At the time of the last follow-up, only nonoperative care had been provided in 18 cases, and no additional symptoms were noted in 17 of these 18 cases. These data offer useful information re- 
garding making informed decisions for patients with RFs after ASD surgery.

\section{References}

1. Akazawa T, Kotani T, Sakuma T, Nemoto T, Minami S: Rod fracture after long construct fusion for spinal deformity: clinical and radiographic risk factors. J Orthop Sci 18:926-931, 2013

2. Barton C, Noshchenko A, Patel V, Cain C, Kleck C, Burger $\mathrm{E}$ : Risk factors for rod fracture after posterior correction of adult spinal deformity with osteotomy: a retrospective caseseries. Scoliosis 10:30, 2015

3. Bess S, Line B, Fu KM, McCarthy I, Lafage V, Schwab F, et al: The health impact of symptomatic adult spinal deformity: comparison of deformity types to United States population norms and chronic diseases. Spine (Phila Pa 1976) 41:224233, 2016

4. Carreon LY, Puno RM, Dimar JR II, Glassman SD, Johnson JR: Perioperative complications of posterior lumbar decompression and arthrodesis in older adults. J Bone Joint Surg Am 85-A:2089-2092, 2003

5. Charosky S, Guigui P, Blamoutier A, Roussouly P, Chopin $\mathrm{D}$ : Complications and risk factors of primary adult scoliosis surgery: a multicenter study of 306 patients. Spine (Phila Pa 1976) 37:693-700, 2012

6. Cho SK, Bridwell KH, Lenke LG, Yi JS, Pahys JM, Zebala LP, et al: Major complications in revision adult deformity surgery: risk factors and clinical outcomes with 2- to 7-year follow-up. Spine (Phila Pa 1976) 37:489-500, 2012

7. DeWald CJ, Stanley T: Instrumentation-related complications of multilevel fusions for adult spinal deformity patients over age 65: surgical considerations and treatment options in patients with poor bone quality. Spine (Phila Pa 1976) 31 (19 Suppl):S144-S151, 2006

8. Gehrchen M, Hallager DW, Dahl B, Harris J, Gudipally M, Jenkins S, et al: Rod strain after pedicle subtraction osteotomy: a biomechanical study. Spine (Phila Pa 1976) 41 (Suppl 7):S24, 2016

9. Hyun SJ, Lenke LG, Kim YC, Koester LA, Blanke KM: Comparison of standard 2-rod constructs to multiple-rod constructs for fixation across 3-column spinal osteotomies. Spine (Phila Pa 1976) 39:1899-1904, 2014

10. La Maida GA, Luceri F, Gallozzi F, Ferraro M, Bernardo M: Complication rate in adult deformity surgical treatment: safety of the posterior osteotomies. Eur Spine J 24 (Suppl 7):879-886, 2015

11. Luca A, Lovi A, Galbusera F, Brayda-Bruno M: Revision surgery after PSO failure with rod breakage: a comparison of different techniques. Eur Spine J 23 (Suppl 6):610-615, 2014

12. Luca A, Ottardi C, Sasso M, Prosdocimo L, Luca A: Instrumentation failure following pedicle subtraction osteotomy: the role of rod material, diameter, and multi-rod constructs. Eur Spine J 26:764-770, 2017

13. Pellisé F, Vila-Casademunt A, Ferrer M, Domingo-Sàbat M, Bagó J, Pérez-Grueso FJS, et al: Impact on health related quality of life of adult spinal deformity (ASD) compared with other chronic conditions. Eur Spine J 24:3-11, 2015
14. Pichelmann MA, Lenke LG, Bridwell KH, Good CR, O'Leary PT, Sides BA: Revision rates following primary adult spinal deformity surgery: six hundred forty-three consecutive patients followed-up to twenty-two years postoperative. Spine (Phila Pa 1976) 35:219-226, 2010

15. Schwab FJ, Hawkinson N, Lafage V, Smith JS, Hart R, Mundis G, et al: Risk factors for major peri-operative complications in adult spinal deformity surgery: a multi-center review of 953 consecutive patients. Eur Spine J 21:2603-2610, 2012

16. Smith JS, Shaffrey CI, Ames CP, Demakakos J, Fu KMG, Keshavarzi S, et al: Assessment of symptomatic rod fracture after posterior instrumented fusion for adult spinal deformity. Neurosurgery 71:862-867, 2012

17. Smith JS, Shaffrey E, Klineberg E, Shaffrey CI, Lafage V, Schwab FJ, et al: Prospective multicenter assessment of risk factors for rod fracture following surgery for adult spinal deformity. J Neurosurg Spine 21:994-1003, 2014

18. Soroceanu A, Diebo BG, Burton D, Smith JS, Deviren V, Shaffrey C, et al: Radiographical and implant-related complications in adult spinal deformity surgery: incidence, patient risk factors, and impact on health-related quality of life. Spine (Phila Pa 1976) 40:1414-1421, 2015

19. Wang H, Guo J, Wang S, Yang Y, Zhang Y, Qiu G, et al: Instrumentation failure after posterior vertebral column resection in adult spinal deformity. Spine (Phila Pa 1976) 42:471-478, 2017

20. Yamanaka K, Mori M, Yamazaki K, Kumagai R, Doita M, Chiba A: Analysis of the fracture mechanism of Ti-6Al-4V alloy rods that failed clinically after spinal instrumentation surgery. Spine (Phila Pa 1976) 40:E767-E773, 2015

21. Yamato Y, Matsuyama Y, Hasegawa K, Aota Y, Akazawa T, Iida T, et al: A Japanese nationwide multicenter survey on perioperative complications of corrective fusion for elderly patients with adult spinal deformity. J Orthop Sci 22:237242,2017

\section{Disclosures}

Drs. Togawa and Oe work at a donation-endowed laboratory in the Division of Geriatric Musculoskeletal Health. The laboratory receives funding from Medtronic Sofamor Danek Inc., Japan Medical Dynamic Marketing Inc., and Meitoku Medical Institution, Jyuzen Memorial Hospital.

\section{Author Contributions}

Conception and design: Yamato. Acquisition of data: Yamato, Hasegawa, Yasuda, Togawa, Yoshida. Analysis and interpretation of data: Yamato. Drafting the article: Yamato. Critically revising the article: Kobayashi, Banno, Oe, Mihara, Matsuyama. Reviewed submitted version of manuscript: all authors. Approved the final version of the manuscript on behalf of all authors: Yamato. Statistical analysis: Yamato.

\section{Correspondence}

Yu Yamato: Hamamatsu University School of Medicine, Hamamatsu City, Shizuoka, Japan. yamato@hama-med.ac.jp. 